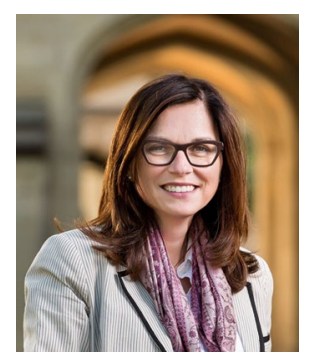

\title{
Not from Venus, not from Mars - all equally superstars
}

\author{
Male immunologists are scientists and nothing more; female immunologists are scientists and \\ nothing less.
}

Credit: Fabienne Mackay

$\Lambda$ sastoc $s$ a successful female immunologist who has reached senior academic executive positions, I am frequently asked how I have managed to avoid the impediments blocking scientific promotion that beset so many of my gender. The answers - as one might expect, given the prevalence of the problem - are not straightforward, and reflect a hardwired, inbuilt bias in the system that has been operational for many decades. As scientists, no one would question the significance of the data that shows dramatically different promotion trajectories for men versus women, inferior grant and fellowship outcomes for women versus men and widely divergent recognition of the scientific outputs of the two sexes by research academies. Over the recent decades we have perhaps made it to the metaphorical basecamp - an achievement in itself. However, from whatever perspective you take, there is still an enormous mountain to climb.

At a personal level, one critical factor for my career development was the guidance from outstanding mentors, who were progressive by the standard of the day (and would still be regarded as progressive in many institutions). These men encouraged and supported me, undeterred by my gender or 'biological responsibilities' and able to see my potential and provide me with pathways to achieve it. These pathways came in the form of strong collaborations, which continued during my pregnancy and maternity leave, partially offsetting the inevitable gap in productivity. They also manifested in repeated opportunities to present my work at international conferences, allowing me to maintain my visibility in the field. This was largely possible because my partner also worked in immunology, and we shared many meetings. For women without that luxury, provision must be made for additional funding to support child care, which will enable travel and presentations at meetings. It is incumbent on existing research leaders to actively identify their future women research leaders and provide them with opportunities for ongoing engagement and research output during periods of personal leave. In the absence of such proactive approaches, the status quo will be maintained.
Now that I am a senior academic executive in a large Australian university, I am more acutely aware, at scale, of the myriad of issues women face throughout their research careers and the dilemmas I face in addressing these challenges. The greatest inequity exists in the recruitment and promotion of women in research. At face value, male applicants often appear to have a superior curriculum vitae, particularly if the section on 'relative to opportunity' is overlooked, as it so often is. We must tackle this if women are to be judged fairly in the advancement stakes. Scientific trajectory prior to leave of absence, for any reason, must be considered (for all genders), and evaluation of output during reduced work periods must be commensurate with circumstances. However, this alone is not sufficient, as it fails to account for reduced opportunities to speak at conferences, which leads to diminished national and international profiles, and this in turn impacts the formation of collaborative links. It's a complex problem, and there is no simple rubric, but giving only cursory attention to this issue will ensure that the existing inequity is not redressed.

In terms of advancement, I am frequently amazed by the number of male applicants who seek promotion well before they meet standard metrics (which is fine, ambition is to be encouraged) compared with the number of women who do so several years later than their achievements would warrant. Where does the problem lie here? In part, it reflects societal values, which see women underpaid and underrecognized. A consistent failure of female peers to be promoted establishes a vicious cycle whereby women undervalue their own worth. Again, the academic leaders of our universities and institutes have a responsibility to rectify this by actively identifying high-performing women (and men) and encouraging them to seek promotion. Collectively, women also need to be more forthright in the promotion stakes and put themselves forward earlier.

In Australia, these issues have been brought sharply into focus with the recent funding round of the National Health and Medical Research Council (NHMRC). Changes to the NHMRC were implemented in 2019, and recent results for the first round of the most competitive award, the Investigator Grant, showed an overwhelmingly high success rate for men compared to women at the more senior level of this award. This sobering result for Australian female researchers will further hinder our ability to recruit and promote funded women to senior levels.

So, what are the solutions? As a leader, it is difficult to repair years of damage done to women researchers by the system, and my responsibility is to recruit the best candidates, as determined by the quality of their research. However, my senior staff and I have decided to stop the ineluctability of this situation at the onset by providing support to our best emerging female research stars in the form of a 'pair of hands' in the laboratory for up to two years during a career disruption. We also provide support for women invited to present their work at a conference so they can bring their children.

For Australian medical research, the paucity of women at senior levels will not change unless we actively implement change, starting with more equitable research funding outcomes. This could be achieved by ranking all grant applicants on the basis of the quality of their research, then separating genders and applying the same success rate to each. Based on history, the overall average score for women will likely be lower than that for men, but I would argue that this reflects unconscious gender bias and unfair weighting for diminished career opportunity, rather than lower-quality applications. This model will ensure research opportunities for greater numbers of women and provide enduring leadership and inspiration for future generations of women scientists.

\section{Fabienne Mackay (iD) 1,2 \\ ${ }^{1}$ Department of Microbiology and Immunology, School of Biomedical Sciences and The Peter Doherty Institute for Infection and Immunity, The University of Melbourne, Melbourne, Victoria, Australia. ${ }^{2}$ Department of Immunology and Pathology, Monash University, Victoria, Australia. e-mail:fabienne.mackay@unimelb.edu.au}

